\title{
¿QUÉ PAPEL JUEGAN LOS BIBLIOTECARIOS EN LAS ALTMETRICS?
}

\author{
Nieves González-Fernández-Villavicencio* \\ Biblioteca de la Universidad de Sevilla \\ María-Isabel Domínguez-Aroca** \\ Biblioteca de la Universidad de Alcalá (Madrid)
}

Antonio Calderón-Rehecho***

Biblioteca de la Universidad Complutense de Madrid

Pablo García-Hernández ${ }^{* * *}$

Biblioteca de la Universidad de Salamanca

\begin{abstract}
Resumen: Se busca identificar los pasos, a modo de hoja de ruta, que deben dar los bibliotecarios en la difusión, formación y asesoramiento de las métricas complementarias o altmetrics en el marco de los servicios que ofrecen a sus investigadores. Así mismo se enumeran una serie de razones por las que los bibliotecarios deben tener un papel activo en relación a estas métricas sin olvidar sus limitaciones. Se ha realizado una revisión de la bibliografía y dirigido una encuesta online a bibliotecarios de universidades españolas para conocer su grado de implicación y actividad en este tema, contribuyendo a proporcionar la visión que tienen de las altmetrics ya que representan un elemento estratégico en su difusión y formación.

Palabras clave: Altmetrics; bibliotecarios; biblioteca universitaria; investigadores; métricas de producción científica; evaluación de la investigación.

Title: WHAT ROLE DO LIBRARIANS PLAY IN ALTMETRICS?

Abstract: Our aim is to identify the steps that librarians can take concerning altmetrics within the framework of the research support services. The current bibliography on altmetrics has been reviewed. A survey has also been carried out of librarians of Spanish universities to find out their degree of involvement in the matter and the setting up of relevant initiatives. Likewise, a thorough study which would enable librarians to find out about the use which their researchers make of social networks and the interest that they may have in altmetrics is needed. This research offers a user guide for librarians as an orientation in their activities and initiatives related to altmetrics, as well as a list of arguments which justify the reasons why librarians should undertake these initiatives. We contribute a view of altmetrics from the perspective of librarians as a strategic element of dissemination and training in altmetrics.

Keywords: Altmetrics; librarians; academic library; researchers; scientific production metrics; research evaluation.
\end{abstract}

\section{INTRODUCCIÓN}

La web social se ha convertido en un medio que permite conocer el impacto de la investigación científica más allá de la información que proporcionan los índices de citas (Borrego, 2014). Existen evidencias de que los investigadores están utilizando los medios sociales para descubrir papers, contactar y comunicarse con otros colegas y difundir sus trabajos y recomendaciones, como demuestran los resultados de un estudio publicado en la revista Nature (Noorden, 2014), aunque es cierto que no utilizan estos medios de forma masiva (You, 2014).

En el Informe Horizon de 2014 dedicado a bibliotecas universitarias, se habla de las altmetrics como una tecnología bibliométrica abierta que se basa en la web social para el análisis de la publicación científica y cuyo uso por parte de las bibliotecas se hará extensivo en dos o tres años (Johson et al., 2014).

Cuando nos referimos a un artículo, con las altmétricas se puede conocer cuántas veces un artículo ha sido visto, descargado, rehusado, adaptado, compartido, marcado como favorito y comentado.

Aunque las incógnitas siguen siendo muchas en relación a cómo interpretar los datos, qué se está midiendo y qué representa, gran parte de las bibliotecas universitarias se están interesando por esta innovadora aproximación para

\footnotetext{
*nievesg@us.es

**misabel.dominguez@uah.es

***acaldero@ucm.es

****.pgh@usal.es
}

Recibido: 11-03-2015; $1^{a}$ versión: 06-05-2015; aceptado: 16-06-2015.

GONZÁLEZ FERNÁNDEZ-VILLAVICENCIO, N.; DOMÍNGUEZ AROCA, M. I.; CALDERÓN REHECHO, A. y GARCÍA HERNÁNDEZ, P. ¿Qué papel juegan los bibliotecarios en las altmetrics? Anales de Documentación, 2015, vol. 18, $\mathrm{n}^{\circ} 2$. Disponible en: Doi: http://dx.doi.org/10.6018/analesdoc.18.2.222641. 
cuantificar el impacto científico de su institución, entendiéndolas como métricas complementarias, más que

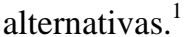

\section{METODOLOGÍA Y OBJETIVOS}

La pregunta que nos planteamos es: ¿cuál es el papel que los bibliotecarios deben jugar en el escenario de las altmetrics como métricas complementarias del impacto de la publicación científica?

Nuestro objetivo es definir cuál debería o podría ser el papel de la biblioteca y del bibliotecario como puente entre las denominadas altmetrics y los investigadores y enumerar las acciones que debería llevar a cabo en forma de hoja de ruta. Para responder a esta pregunta, se hace una investigación bibliográfica que aporta el fundamento teórico, el estado de la cuestión y la identificación y descripción de los principales agentes involucrados. Este estudio, y la experiencia profesional de los autores, nos llevan a plantear las razones por las que los bibliotecarios deberían incorporarlas en su actividad diaria y los pasos a seguir, mostrando prácticas y ejemplos de bibliotecas donde ya lo han hecho.

Con el fin de aportar datos hemos realizado una pequeña encuesta online dirigida a los bibliotecarios de universidades españolas. El reducido número de respuestas no representa a la totalidad de la población por lo que los resultados no pueden hacerse extensivos a todos los bibliotecarios españoles pero permite ofrecer un panorama general, aunque no exhaustivo, de la situación en las bibliotecas universitarias españolas respecto a esta cuestión. Pensamos que se trata de una primera aproximación a las prácticas con altmetrics desde las bibliotecas españolas.

Para la realización de la encuesta, se han seleccionado preguntas que dieran una visión general desde el punto de vista de los bibliotecarios, referidas a su nivel de conocimiento y formación en este tema y el de sus investigadores, nivel de difusión desde las sedes web de sus bibliotecas y productos dirigidos a los investigadores, nivel de responsabilidad de los bibliotecarios en la difusión y formación de altmetrics y tareas que se estén llevando a cabo.

Para terminar se presentan las conclusiones del trabajo realizado.

\section{RESULTADOS: ESTADO DE LA CUESTIÓN Y ACCIONES}

Los resultados obtenidos incluyen el estado de la cuestión de las altmetrics, la identificación de los agentes involucrados, iniciativas de referencia en bibliotecas y la opinión de los encuestados, que nos llevan a proponer 10 razones por las que los bibliotecarios deben tomar parte en la difusión y formación de las altmetrics así como 10 pasos que deben dar.

\subsection{La actualidad de altmetrics a partir de la bibliografía}

Hasta este momento las métricas tradicionales han servido a la comunidad científica para medir el impacto de la investigación, pero en los últimos años han mostrado su falta de adaptación al nuevo ecosistema académico, ya que son difíciles de aplicar a resultados de investigación comunes, como informes técnicos, documentos de trabajo, datasets, software, presentaciones en conferencias o productos multimedia (Konkiel, 2013).

El deseo de encontrar métricas alternativas es un síntoma de que algo no va bien en la evaluación de la investigación, ${ }^{2}$ lo que lleva a preguntarse ¿'son sólo las citas los elementos que deben medirse?, ¿no importan las lecturas reales que asientan investigaciones posteriores?, ¿cómo se miden estas lecturas?, ¿con descargas, accesos, recomendaciones?, ¿qué ocurre con aquellas que no se reflejan en la producción científica pero que triunfan en los ámbitos de la formación, incluso en la que forma nuevos investigadores?, ¿qué con las que están fermentando, sobre las que todavía no se puede publicar por unas u otras razones?

En este contexto surge el concepto de altmetrics, como el estudio y uso de las métricas del impacto científico que se basan en la actividad de herramientas y entorno online (Priem, Groth y Taraborelli, 2012), o como lo definen Galloway et al. (2013) “Altmetrics are the tools that help track a scholar’s influence and relevance beyond traditional citation metrics. Altmetrics provide immediate feedback because they rely on real-time data and interactions and can be quantified quickly”.

Las métricas alternativas surgen en un momento en que tanto autores como editores empiezan a comprender que las métricas tradicionales no son suficientes para conocer el interés que despiertan sus publicaciones. Plantean a su vez un cambio en la concepción del impacto científico, poniendo el foco de atención tanto en el artículo propiamente dicho 
como en los productos de investigación derivados del mismo. Detectan diferentes tipos de impacto por lo que proporcionan a los científicos nuevos filtros para estar al día de las publicaciones con mayor impacto social en sus campos de investigación.

En los últimos tiempos el interés por las métricas alternativas ha crecido de forma fulgurante y se ve reflejado en la aparición de un número cada vez mayor de publicaciones científicas que tratan de ponerlas en contexto.

Tras una primera fase de escepticismo se ha aceptado la invitación planteada en el Manifiesto Altmetrics (Priem, et al., 2012), -génesis de este nuevo enfoque- y gran cantidad de investigadores especializados en el campo han tratado el tema. La adecuación del término altmetrics para definir las nuevas métricas ha sido puesta en duda por diversos autores: Todd Carpenter (2015) afirma que no deben considerarse ya métricas alternativas debido a su consolidación como “estándar” en la comunidad científica, mientras que Ronald y Fred (2013) proponen la acepción influmetrics como más apropiada.

Los enfoques han sido diversos pero existen unas corrientes principales: 1) altmetrics como indicador o indicio de calidad de la investigación; 2) modo en que utilizan los investigadores la web social; 3) facilidad para medir el impacto social de la investigación; 4) correlación entre altmetrics y métricas tradicionales; 5) uso de métricas alternativas en repositorios institucionales y relación con Open Access.

Las principales críticas hacia las altmetrics se han centrado en los escasos indicios sobre su utilidad en el contexto académico, lo que lleva a la necesidad de evaluarlas (Sud y Thelwall, 2014). En este sentido, Torres-Salinas y Cabezas-Clavijo (2013) señalan las dudas existentes sobre su validez estadística así como sobre el significado de sus resultados. Por otra parte, la posible manipulación de resultados que puedan tener las nuevas métricas suponen una preocupación en la comunidad científica, ante la cual los proveedores de datos altmétricos están trabajando para mejorar sus herramientas y detectar cualquier actividad sospechosa (Adie, 2013).

Las revistas científicas especializadas en temas bibliométricos son las que más interés han puesto en la materia. Más de un centenar de citas ha obtenido el Manifiesto (Priem et al., 2012) así como las publicaciones de los prolíficos fundadores de la herramienta ImpactStory ${ }^{3}$.

Sin embargo, la conversación sobre altmetrics se encuentra actualmente en lugares tradicionalmente no pertenecientes al contexto académico (Galligan y Dyas-Correia, 2013) como la web. Así, además de los más extendidos blogs científicos, páginas web de todo tipo y redes sociales propiamente dichas se han hecho eco del nuevo hot topic bibliométrico (Chia y Wee, 2014). La irrupción de las altmetrics ha generado revuelo en la comunidad científica e interés en el público general y su impacto es tanto académico como popular (Konkiel y Scherer, 2013).

El autor con más publicaciones es Mike Thelwall ${ }^{4}$ ( $H$ index Scopus=40), profesor de la Universidad de Wolverhampton que encamina su investigación al estudio del big data. En la red social Twitter, podemos encontrar a otros influencers en altmetrics como Keita Bando (@KeitaBando) (klout=46), investigador japonés que dirige la organización sin ánimo de lucro MyOpenArchive; o Todd Carpenter (@TAC_NISO) (klout=57), director ejecutivo de NISO (National Information Standards Organization).

Por otro lado, desde el año 2011 numerosos eventos académicos han tratado en sus programas las métricas alternativas, con preferencia por el formato de workshop, destacando los celebrados en el marco de la Association for Computing Machinery (ACM) Web Science Conference y los organizados por The Alfred P. Sloan Foundation y PLOS. Hasta 2014 no se celebró un encuentro completamente dedicado a las nuevas medidas de evaluación del impacto académico, el 1st Altmetric Conference (London) ${ }^{5}$ que contó con la participación de Altmetric.com y editoriales como Springer, Elsevier o Thomson Reuters.

\section{2 ¿Quiénes son los agentes involucrados en las altmetrics?}

Los actores afectados, con un papel que interpretar en estas nuevas métricas, son: 1) los proveedores; 2) los editores, revistas científicas y empresas y organizaciones que miden el impacto de la investigación con métricas alternativas; 3) los repositorios; 4) los investigadores y 5) los bibliotecarios.

\section{1) Los proveedores}

La información proporcionada por las métricas alternativas se obtiene en virtud del desarrollo de algunas herramientas cuyos proveedores dejan en muchos casos sus APIs (Application Programming Interface) o plugins en abierto. 
- PLoS Article-Level Metrics application (ALM). Proporciona un conjunto de métricas alternativas al impacto tradicional del artículo y mide el rendimiento general y alcance de los artículos de investigación publicados por PLOS ${ }^{6}$. Su API está disponible de forma libre. PLOS cuenta con Altmetrics Collection cubriendo la identificación de los sesgos en las mediciones y la validación de modelos de descubrimiento científico / recomendación basada en altmetrics.

- Plum Analytics <http://www.plumanalytics.com/>, servicio de pago propiedad de EBSCO, se comercializa para universidades e instituciones de investigación (González-Fernández-Villavicencio, 2014). Anunció la apertura de su API en octubre de 2013. ${ }^{7}$ Mide artículos, capítulos de libros, libros, ensayos clínicos, conjuntos de datos, figuras, subvenciones, patentes, presentaciones, código fuente, vídeos; lo que es su principal ventaja respecto a sus competidores (Peters et al., 2015). Los datos que proporciona son descargas, vistas, explotaciones de libros, préstamo interbibliotecario, entrega de documentos; datos de captura de favoritos, marcadores, lectores, grupos; menciones de blogs, noticias, artículos de Wikipedia, comentarios, críticas; de medios sociales, tuits, me gusta; citas del Web of Science, Scopus, Google Scholar, Microsoft Academic Search. Consigue que los perfiles de los investigadores se conecten con sus identificadores ORCID (Open Researcher and Contributor ID) cuando la institución se convierte en cliente de PlumX.

- ImpactStory <https://impactstory.org/>, es una aplicación basada en web que facilita el seguimiento del impacto de la investigación financiada por la National Science Foundation y la Alfred P. Sloan Foundation. En la actualidad es de pago, pero sin ánimo de lucro. El software es de código abierto bajo una licencia MIT (Massachusetts Institute of Technology). Dirigido al uso individual de investigadores, hace seguimiento de DOIs (Digital Object Identifier), PubMedID, URLs, Slideshare, Github y Dryad. Recoge descargas en pdf, html, vistas de PLOS y puede importar citas de Google Scholar, ORCID, Figshare, GitHub, Slideshare, Wikipedia (desde febrero de 2015) ${ }^{8}$ y otras fuentes. Los usuarios finales pueden acceder a los perfiles gratuitamente. Dejó de ofrecer datos mediante API y ahora solo lo hace a través de perfiles privados en su propia red.

- Altmetric <http://www.altmetric.com/>, recoge las métricas a nivel de artículo proporcionadas por menciones de agencias de noticias (inglés solamente), blogs, tweets, Facebook, Wikipedia, Pinterest, Google+, YouTube, grupos de Linkedin, Reddit, YouTube, F1000, PubPeer y Publons, etc.; lectores de Mendeley, CiteUlike, Connotea. Tiene una versión "Freemium" que permite un uso limitado de la API y un servicio completo de su API e informes. Con su famoso donut para WordPress ${ }^{9}$ el botón Bookmarklet $^{10}$, denominado Altmetric it! para los navegadores Chrome, Firefox y Safari, permite obtener de forma instantánea métricas a nivel de artículo para cualquier trabajo reciente, de forma gratuita. Funciona en páginas que contienen DOIs, como PubMed IDs y ArXiv IDs. Cuenta con una galería de APIs y conectan con ORCID a través de la app. Ha creado también una versión de Altmetrics para investigadores. ${ }^{11}$

Existen otras herramientas que hacen un seguimiento de las menciones a artículos o citas en línea, como CitedinAPI ${ }^{12}$ en blogs, bases de datos y wikis (como Wikipedia) en base al identificador de PubMed; ReaderMeter, un mashup que obtiene los datos a nivel de autor y de artículo a través del API de Mendeley; PaperCritic que ofrece a los investigadores una forma de observar todo tipo de comentarios sobre su trabajo científico y revisar fácilmente el de los demás o el propio Mendeley, que ofrece estadísticas del número de lectores de los artículos que recoge, y que son reutilizados a través de su API por el resto de proveedores. En este punto se puede incluir también Webometric Analyst, un software de webmetría que, entre otros, analiza datos procedentes de Twitter, Mendeley, Altmetric.com o ArXiv.

\section{2) Editores y revistas científicas}

Entre los editores que proporcionan datos altmétricos, destaca Public Library of Science (PLOS), que fue el primero en proporcionar este tipo de datos de sus publicaciones a través del mencionado ALM. Le han seguido otros grandes editores como el Grupo Editorial Nature, Springer, Elsevier, y más recientemente Wiley. Elsevier ha lanzado The Metrics Development Program ${ }^{13}$ cuyo punto 2 incorpora las altmetrics. HighWire, ofrece como opcional Article Level Metric (ALM) para los editores que utilizan su plataforma y más de 15 editores de suscripción participan en KUDOS $^{14}$, nuevo servicio gratuito que se puso en marcha a finales de abril de 2014 para ayudar a los investigadores y sus instituciones a maximizar la visibilidad e impacto de sus artículos científicos publicados, incluyendo indicadores de descargas, citaciones y métricas alternativas. BioMedCentral, ha incorporado la utilidad Altmetric.com para 
analizar la repercusión de sus publicaciones en las redes sociales. Se trata de una información que aparece en los detalles del artículo, junto con las métricas tradicionales.

Elsevier también participa en el proyecto NISO Altmetrics ${ }^{15}$ para explorar, identificar y avanzar en "normas" y "buenas prácticas"16 y mejorar los métodos de evaluación de la comunidad académica.

SPARC, utiliza las ALM ${ }^{17}$ de PLOS, sobre lo que publicó un estudio (Tananbaum, 2013).

A pesar del interés de empresas y organizaciones aún queda mucho por hacer en normalización, transparencia, cobertura temporal, etc. (Zahedi, Fenner y Costas, 2014). Las últimas investigaciones señalan que por ahora los datos de investigación no son ampliamente compartidos en los medios sociales; sin embargo, el mayor uso y adopción de DOI y otros identificadores permanentes está permitiendo aumentar la visibilidad de los datos de investigación y la inclusión de herramientas altmétricas (Peters, et al., 2015).

Por su parte, las revistas científicas están incorporando las puntuaciones altmétricas de forma individual o beneficiándose de las plataformas de bases de datos de las que forman parte. Por ejemplo un artículo publicado en New England Journal of Medicine el 21 de noviembre de 2013, obtuvo una calificación Altmetric.com de 1.837 y otro publicado el 8 de noviembre en PLOS ONE, 960. ${ }^{18}$

En noviembre de 2013 una iniciativa piloto de la empresa Altmetric.com, incluyó datos altmétricos a nivel de artículo en 26 revistas de ScienceDirect ${ }^{19}$ entre ellas The Lancet, Neuron o American Journal of Preventive, de Elsevier. La misma empresa proporciona los 100 principales artículos anuales. ${ }^{20}$

Las revisiones Cochrane y Wiley se han asociado con Altmetric.com, para rastrear el impacto de los artículos académicos y conjuntos de datos en los medios de comunicación tradicionales y sociales. Las puntuaciones altmétricas e insignias se mostrarán en cada protocolo de Cochrane y en la base de datos Cochrane de revisiones sistemáticas. ${ }^{21}$

\section{3) Los repositorios}

Respecto a los repositorios, es de destacar la estrecha relación entre altmetrics y el movimiento de acceso abierto a la ciencia. Es una oportunidad para autores, editores y gestores de repositorios poder obtener nuevos datos además de las estadísticas de uso y descarga. Por ello, repositorios de todo el mundo están incorporando altmetrics para hacer un seguimiento del uso e intercambio de la producción científica en la web social. Para Konkiel y Scherer (2013): "University administrators are increasingly trying to find new ways to measure the impact of the scholarly output of their faculty, students and researchers through quantitative means. By reporting altmetrics (alternative metrics based on online activity) for their content, institutional repositories can add value to existing metrics - and prove their relevance and importance in an age of growing cutbacks to library services”.

En España fue Digital.CSIC en 2013, el primer repositorio que enriqueció las estadísticas de uso con datos altmétricos (CSIC Abierto, 2013). Otros repositorios que las han ido incorporando han sido el repositorio de la Universidad de Alcalá ${ }^{22}$ o el de la Universidad Politécnica de Cataluña ${ }^{23}$ en 2014.

En enero de 2014, el ranking web de repositorios anunciaba que había incluido indicadores altmétricos de las siguientes fuentes: Academia.edu, Facebook, Linkedin, Mendeley, ResearchGate, Slideshare, Twitter, Wikipedia y YouTube. $^{24}$

\section{4) La comunidad científica}

La web social ha dado la oportunidad a los investigadores de crear, anotar, reutilizar y representar información de un modo difícil de imaginar hace unos años (Procter et al., 2010). Cada vez más los investigadores y grupos de investigación van teniendo una presencia más importante en las redes sociales como Twitter, Facebook, Google+, ResearchGate, Academia.edu, etc. Dicha presencia es fundamental para lograr datos altmétricos fiables.

Algunas universidades están dándose cuenta de las posibilidades de los datos altmétricos. La Universidad Rovira i Virgili ${ }^{25}$ por ejemplo, difunde en sus noticias que entre su personal investigador se halla el segundo artículo científico de mayor impacto mundial en las redes sociales en 2013 según el ranking de Altmetric.com. 
En Gran Bretaña, los científicos están autorizados a utilizar altmetrics para demostrar el impacto social en los informes para el REF (Research Excellence Framework) cuya evaluación influye en la financiación, señala Graeme Rosenberg, gerente del REF en el Higher Education Funding Council de Bristol (Kwok, 2013).

Ante este panorama, NISO consciente de la problemática y la necesidad de recuperar los datos de forma normalizada, comenzó en 2013 un proyecto (NISO, 2014) para desarrollar estándares y buenas prácticas de altmetrics, ya que entre otras ventajas, podría ser potencialmente útil para la evaluación del profesorado y el proceso de acreditación. Indudablemente será un paso decisivo y un punto de inflexión en la puesta en valor de estas métricas alternativas, sobre todo si es adoptada de forma institucional.

\section{5) Los bibliotecarios}

Los bibliotecarios de bibliotecas universitarias y especializadas, gestionan, organizan, forman e informan usualmente sobre citas e impacto a sus investigadores. Por eso la Association of College \& Research LIbraries (ACRL, 2014) afirma que los bibliotecarios universitarios poseen la cualificación necesaria para un uso apropiado de las altmetrics y promover entre la comunidad académica el impacto y valor de la investigación producida en sus instituciones. Así, los bibliotecarios deberían formar e informar sobre estas métricas alternativas como un servicio más de la biblioteca, al estar "bien posicionados para informar y apoyar a investigadores y gestores en su uso" (Lapinski, Piwowar y Priem, 2013).

Además, los recursos suscritos por la biblioteca están incorporando las altmetrics, tanto en los repositorios (Konkiel y Scherer, 2013), como en las bases de datos y recursos electrónicos: el API de PlumX se ha integrado en OCLCWordCat Search $^{26}$ y se están desarrollando plugins para las distintas plataformas de recuperación de la información, como el gratuito de Primo creado por Almetric.com. ${ }^{27}$

Seguidamente se exponen las razones por las cuales los bibliotecarios deberían o podrían incorporarlas en su actividad diaria y los pasos a seguir para poder lograrlo, mostrando posteriormente prácticas y ejemplos de bibliotecas del mundo que las han incorporado.

\subsection{0 razones para que los bibliotecarios incorporen las altmetrics en su actividad diaria}

Los bibliotecarios juegan un papel clave en el área de las métricas alternativas como asesores, formadores y defensores en temas de publicación científica, tal y como plantea el citado informe ACRL (2014) sobre tendencias en bibliotecas universitarias o Merlo-Vega (2011).

En el contexto de la web social y la ciencia 2.0, las altmetrics están vinculadas a las tareas del bibliotecario de apoyo a la investigación y a la figura del bibliotecario temático o integrado en la docencia y la investigación (González-Fernández-Villavicencio, 2013). Para Sutton (2014) las altmetrics son la extensión natural de lo que las bibliotecas y bibliotecarios ya hacen. No en vano PlumX fue fundado en 2012 por un bibliotecario y un tecnólogo.

El Libro blanco sobre altmetrics (NISO, 2014) dice que "bibliotecas y bibliotecarios poseen la cualificación necesaria para formar y difundir el uso apropiado de altmetrics para promover a la comunidad académica global, el impacto, la atención y el valor de la investigación producida en sus instituciones”. Tanto NISO (2014) como ACRL (2014) ven indicios de que las bibliotecas están incorporando información sobre altmetrics al incluir en las guías por materias los recursos para la comunicación científica, el impacto y la gestión de citas. Autores como Bando (2013) se preguntan cuándo los bibliotecarios van a incluir las métricas alternativas en su tarea diaria. Sin embargo esto no quiere decir que los bibliotecarios deban entregarse ciegamente a la difusión de estas métricas sin plantear seriamente sus limitaciones (Barnes, 2015).

Consideramos que las altmetrics son de interés para los bibliotecarios desde varios puntos de vista:

1. Como bibliotecarios de apoyo a la investigación son colaboradores asesores que ayudan a los investigadores a conocer, mejorar, comprender y construir su impacto científico, en tiempo real, demostrando el valor de sus investigaciones y la atención que obtienen. Varios autores afirman que estas prácticas convierten al bibliotecario en un auténtico especialista en el proceso de la comunicación científica (Priem, Piwowar y Hemminger, 2012; Galligan y Dyas-Correia, 2013; Lapinski, Piwowar y Priem, 2013) ya que evidencian la posible calidad y utilidad de facetas académicas de las que no se tenía información con las métricas tradicionales (ACRL, 2014). Esta información puede ser importante para la promoción y acreditación de los profesores investigadores, para identificar tendencias emergentes en la investigación y obtener financiación para proyectos. La implicación y saber hacer de los 
bibliotecarios en esta tarea les garantiza un papel estratégico en el proceso de producción y comunicación científica académica (Holmes, 2014).

2. Como bibliotecarios que monitorizan el impacto de la actividad científica de su institución, demostrando el ROI de la inversión pública en la educación superior (Sutton, 2014). Altmetric.com ha anunciado la edición institucional de su producto ${ }^{28}$ para ofrecer a bibliotecarios, gestores de investigación e investigadores una forma fácil e intuitiva de monitorizar y registrar el impacto online de las publicaciones y artículos científicos de los miembros de la institución, rentabilizando los escasos recursos de la biblioteca y su institución. Los gestores universitarios pueden presentar resultados suplementarios a las métricas tradicionales a los stakeholders internos y externos, para demostrar el valor de la institución con diferentes objetivos, como identificar tendencias ${ }^{29}$ o buscar financiación (Sutton, 2014).

3. Como asesores institucionales en temas de producción científica. Los gestores universitarios están demandando que los bibliotecarios se pronuncien sobre las métricas alternativas, o sobre la compra de productos que los incluyan (Delasalle, 2014), lo que constituye una oportunidad para formar en sus fortalezas y debilidades a los gestores universitarios e investigadores (Roemer y Borchardt, 2013). Así mismo, los bibliotecarios pueden recomendar a los comités editoriales de las revistas académicas que acepten evidencias de métricas alternativas en su política editorial (Lapinski, Piwowar y Priem, 2013) y ayudar a entender las limitaciones de las altmetrics y cómo compararlas con otras medidas (Brigham, 2014).

4. Como formadores en competencias informacionales y científicas y en sistemas alternativos de la ciencia, las competencias de comunicación científica están teniendo cada vez más relevancia en los planes formativos de las bibliotecas (González-Fernández-Villavicencio, 2013). Con la formación en los conceptos de ciencia 2.0, las bibliotecas incluyen plataformas que permiten compartir libremente todo tipo de información, herramientas de gestión, almacenamiento y networking en el área científica. Todos esos medios generan estadísticas de la actividad e interacción entre sus usuarios en medios sociales, en gestores de referencia, en blogs científicos, en repositorios, etc. Estas métricas afectan a cualquier tipo de material científico y académico, pero no olvidemos que al incluir la formación en sistemas alternativos, hay que abordar también sus limitaciones (Galligan y Dyas-Correia, 2013).

Davis-Kahl, Fishel y Kaye (2014), ofrecen ejemplos de bibliotecas universitarias que han integrado estas competencias informacionales y científicas.

5. Como formadores en el uso de estas nuevas métricas dentro de las competencias científicas específicas de los investigadores (Roemer y Borchardt, 2012; Roemer y Borchardt, 2013) y fomentando la experimentación con las herramientas emergentes (Lapinski, Piwowar y Priem, 2013). Los investigadores se han dado cuenta de la existencia de botones y símbolos con sistemas de ranking que aparecen incrustados en los artículos y repositorios, en las bases de datos y piden información a la biblioteca. En 2014, EBSCO adquiere PLUM Analytics, Springer incluye Altmetric.com en SpringerLink, Wiley añade Altmetric.com a sus revistas en acceso abierto ${ }^{30}$ y Scopus estadísticas de lectura en Mendeley y Altmetric.com ${ }^{31}$. Es una oportunidad para ofrecer guía y consejo en las sesiones de formación (Konkiel y Scherer, 2013).

6. Como seleccionadores de recursos de información para el desarrollo de las colecciones, al tener en cuenta el impacto de las publicaciones. La adquisición de estas altmetrics ofrece una información extra a los bibliotecarios e investigadores sobre citas y usos complementarios de los recursos (descargas, favoritos, menciones, recomendaciones, etc.), además de los datos que ofrecen estadísticas tradicionales como COUNTER (Counting Online Usage of Networked Electronic Resources) ya utilizadas para conocer el uso de las colecciones de recursos electrónicos (Sutton, 2014).

7. Como fuente de información para conocer las necesidades e intereses de sus usuarios (Borrego, 2014). Altmetrics es una extensión natural de lo que los bibliotecarios ya hacen: monitorizar el comportamiento de los usuarios en relación a sus colecciones, sobre todo las electrónicas, para la toma de decisiones (Galligan y DyasCorreia, 2013, p. 58).

8. Para enriquecer colecciones y repositorios. El valor de altmetrics en los repositorios es claro, pues ayuda a tener una visión del conjunto de la colección y anima a depositar más materiales. Puede utilizarse para persuadir e incentivar a potenciales depositantes en acceso abierto. En este sentido habrá que decidir si para la inclusión de estas nuevas métricas en los repositorios se han tenido en cuenta los costes del servicio, las necesidades de soporte técnico, las restricciones de las plataformas y el interés real de los usuarios. 
Además de los servicios altmétricos más conocidos, hay otras formas de implementar estas utilidades a través de APIs y herramientas en OA (Open Access) como ALM de PLOS, servicios y sitios web que trazan el impacto de las publicaciones científicas a través de varios puntos de su ciclo de vida científico, desde la lectura a las revisiones por pares tras la publicación (Scopus, Mendeley, PubMed, Faculty 1000, blogs y redes sociales).

El uso de los repositorios fuera de la academia se incrementa al incluir el impacto de cada material depositado, de qué forma se usa y comparte el contenido (Konkiel y Scherer, 2013; Delasalle, 2014). La institución puede utilizarlo para realizar actividades de divulgación del repositorio y de su producción científica y como medida complementaria de impacto por los comités de evaluación.

9. Como bibliotecarios/investigadores que son, deben promocionar y difundir sus destrezas en el uso y práctica de las altmetrics, conociendo las herramientas de primera mano y usándolas para el impacto de sus propias publicaciones (Roemer y Borchardt, 2013; Lapinski, Piwowar y Priem, 2013), incluyéndolas en sus CV (Curriculum Vitae). ${ }^{32}$

10. Para servir de puente con los proveedores. Los bibliotecarios han estado siempre atentos a las métricas de evaluación de la publicación científica y pueden servir de enlace entre las necesidades de los investigadores e instituciones y los proveedores de las métricas alternativas para indicar los fallos que existen.

\subsection{0 pasos que los bibliotecarios deben dar para incorporarlas}

En líneas generales, la mayoría de los autores están de acuerdo en que los bibliotecarios deben involucrarse en las métricas alternativas ofreciendo su apoyo en tres direcciones: ofreciendo información sobre las últimas tendencias en investigación emergente, apoyando la experimentación con herramientas innovadoras e involucrándose en la formación y resultados de las métricas alternativas (Lapinski, Piwowar y Priem, 2013).

Proponemos los siguientes 10 pasos que deben dar los bibliotecarios:

1. Formarse en métricas alternativas (Lapinski, Piwowar y Priem, 2013). Conocer de primera mano los servicios y herramientas que ofrecen y experimentar con ellos; crearse perfiles y conocer sus funcionalidades, como Altmetric Explorer. Tanto Altmetric.com, ImpactStory como Plum Analytics ofrecen actualmente la posibilidad de acceder a cuentas gratuitas para bibliotecarios.

2. Usar estas herramientas en sus propios perfiles y CV como investigadores. Ponemos como ejemplo la API que genera los datos de investigador en Altmetric en base al perfil ORCID como señalamos en el punto 3.

3. Contactar con otros servicios universitarios de apoyo a la comunicación científica como los servicios de comunicación académicos o los community managers a nivel de universidad, facultad o grupo de investigación así como con los propios investigadores para iniciar proyectos de colaboración para el estudio e implementación de las altmetrics.

4. Asesorar sobre su uso a la institución y difundir sus ventajas y también sus limitaciones. Recomendar a los comités que valoren la concesión de financiación y a los equipos editoriales de las revistas que acepten las diversas métricas de impacto incluyendo enunciados como "Se aceptan evidencias de formas alternativas de impacto”.

5. Formar a usuarios de cualquier tipo en competencias científicas junto a las informacionales, incluyendo formación en gestores de referencias bibliográficas, marcadores sociales, redes sociales, OA, sitios para compartir materiales, etc. (Lapinski, Piwowar y Priem, 2013). Incorporarlos a los materiales formativos, a sus guías por materias.

6. Formar a sus investigadores en competencias científicas incluyendo las métricas alternativas, experimentando con ellas. Ayudar a los investigadores a posicionar sus artículos de forma óptima ante estos servicios, depositando una copia de todos los resultados de su investigación, incluyendo códigos y datos en los repositorios adecuados (Gunn, 2014). Incluso se están incorporando a los CRIS, como en Sympletic Pure o CONVERIS ${ }^{33}$. Incluir indicadores altmétricos en el CV y tener en cuenta a los investigadores en la evaluación de la investigación. Alentar a los investigadores a contar sus propias historias de impacto en sus currículos. Elsevier en concreto recomienda que los bibliotecarios den una serie de pasos como muestra en su conocida infografía. ${ }^{34}$ 
7. Incorporar las altmetrics en los procedimientos de selección de información y de formación de las colecciones. Por ejemplo, ya hay app de Altmetrics para ExlIbris Primo ${ }^{35}$, lo cual implica que además de las estadísticas COUNTER habituales se pueden usar los datos altmétricos para mantener o dejar de suscribir un recurso.

8. Implementarlo en los repositorios, bases de datos, catálogos de las bibliotecas, etc. (Lapinski, Piwowar y Priem, 2013). Son varios los repositorios institucionales universitarios que incorporan las altmetrics, como por ejemplo el de la Universidad de Indiana, IUScholarWorks Repository ${ }^{36}$ y los españoles ya citados. Se recomienda que si no hay actividad no aparezca el botón junto al artículo y el autor debería decidir si quiere que se incluya o no en sus materiales (Delasalle, 2014).

9. Incorporarlo a los procedimientos de estudio de usuarios (detección de sus necesidades).

10. Difundir el uso de estas métricas, sus ventajas y limitaciones, así como la forma de acceder a estos servicios a través de las tecnologías móviles y aplicaciones.

\subsection{Iniciativas de referencia en bibliotecas}

La realidad es que los bibliotecarios manifiestan distintas posturas aunque se busca una colaboración estrecha con los investigadores y el proceso de investigación, en algunos casos propiciada por políticas universitarias de mayor rango, o por consorcios. ${ }^{37}$

Uno de los usos más extendidos es la creación de guías ${ }^{38}$ con formatos diversos y extendidas por todo el mundo, que recopilan información pertinente, explican cómo usarlas, cuáles son los pros y contras, cuáles las fuentes, algunos de los ejemplos más significativos, etc. También han creado páginas web (las libguides lo son) o blogs ${ }^{39}$ específicos donde se habla de ellas, del mismo modo que incluyen entradas en sus blogs generales, organizan $\operatorname{congresos}^{40} \mathrm{o}$ les dedican una sección dentro de ellos. ${ }^{41}$ Las altmétricas se han incluido en la formación sobre evaluación de la actividad investigadora o se plantea como formación en exclusiva. ${ }^{42}$

No sólo a nivel de bibliotecas individuales, también consorcios, o grandes asociaciones publican estados de la cuestión o datos clave (Roemer y Bonchardt, 2014; Sutton, 2014; King y Thuna, 2013). Se apuntan grandes proveedores $^{43}$ que seguramente tienen un interés pero que no dejan de analizar el posible papel de los bibliotecarios que suelen ser sus intermediarios directos.

Algunos ejemplos de buenas prácticas en las que las bibliotecas están experimentando con estas herramientas de bajo riesgo, son:

1.- El University of Pittsburgh Library System está colaborando con Plum Analytics para encontrar formas de evaluar el impacto de la investigación de su universidad a través de medios como repositorios institucionales y medios sociales (Rodgers y Barbrow, 2013). En 2012 creó un directorio con todos sus investigadores con el objetivo de mejorar sus perfiles ${ }^{44}$ y difunde el uso de estas herramientas en su comunidad.

2.- P. Scott Lapinski (bibliotecario de apoyo a la investigación de Harvard Medical School) ofrece formación y asesoramiento a sus usuarios en el uso de altmetrics (Rathemacher, 2014).

3.- Lisa Palmer, bibliotecaria del repositorio institucional Lamar Soutter Library, promociona Altmetric.com en el repositorio eScholarship@UMMS. Para demostrar el impacto a nivel de artículo, ha incluido la API de Altmetric.com en la revista The Journal of eScience Librarianship ${ }^{45}$. El donuts de Altmetric.com aparece en cada artículo con DOI. Ofrece también cursos formativos sobre Altmetrics y guías por materias (LibGuide) en acceso abierto. Ha creado perfiles en ImpactStory para un grupo de investigadores que usan como ejemplo (Rathemacher, 2014).

4.- John Furfey, de la MBLWHOI Library, incorporó altmetrics en el sistema de perfiles de investigadores y sus publicaciones (Bibapp project) de la Woods Hole Oceanographic Institution. A través de una API, incluyeron las métricas de Altmetric.com e ImpactStory en el sistema. ${ }^{46}$ Una de las grandes ventajas es agrupar tendencias en las publicaciones y ofrecer las de los grupos de investigación ${ }^{47}$ que usan los departamentos para decidir las líneas de investigación. Así los bibliotecarios se han convertido en asesores de los investigadores (Rathemacher, 2014).

5.- En los últimos siete años, la biblioteca de the University of New South Wales (UNSW) ha ofrecido un servicio a su comunidad de investigadores denominado RIMS (Research Impact Measurement Service). Tiene como objetivo 
dar soporte a los resultados de la investigación midiendo el impacto de los autores individuales y de los departamentos. Actualmente incluye formación en herramientas altmétricas (Drummond, 2014).

6.- Lucy Ayre es gestora del repositorio institucional LSE Research Online y trabaja con Research Support Services at LSE Library. En una entrevista ${ }^{48}$ afirmaba que recogían estadísticas de descargas, que han añadido una opción para medir cómo está siendo usado en el ámbito social y un badge en todos los documentos con DOI que ayude a los autores a seguir el impacto en línea.

\subsection{Situación en las bibliotecas españolas. Resultados de la encuesta}

A nivel español se observa a través de las páginas webs oficiales que las bibliotecas dedican algunos espacios tanto en la web como en blogs institucionales ${ }^{49}$ y/o dan formación ${ }^{50}$, normalmente incluida en el ámbito general de la evaluación de la actividad investigadora ${ }^{51}$, del mismo modo que informan de estas opciones cuando asesoran a los investigadores.

Uno de los referentes de formación en altmetrics en España es el grupo EC3 ${ }^{52}$ cuyas actividades se dirigen tanto a universidades como bibliotecas universitarias. En algunos de sus Seminarios (como en el IV sobre altmetrics) han participado bibliotecas universitarias y especializadas.

Algunas bibliotecas manifiestan que están preparándose para poder dar una respuesta oportuna y/o están recibiendo información de iniciativas que intentan comercializarse, como PlumX que ofrece la creación de perfiles individuales de investigadores significativos para que las instituciones valoren la oportunidad de ampliarlos a un número más alto de investigadores. También, según se refleja en la encuesta que hemos realizado y de la que hablaremos más adelante, en algún caso han pedido a Altmetric.com una prueba para incluir en el CRIS (Current Research Information System) institucional los datos referidos a sus investigadores.

De cualquier forma, la sensación es que mientras no haya un reconocimiento real los investigadores no van a prestarle demasiada atención. Quizás sea más demandado en el caso de investigadores noveles que necesitan saber si sus publicaciones son descargadas, enlazadas en gestores o más conocidas y con posibilidades de ser citadas.

Con el fin de aportar datos de primera mano a esta investigación sobre bibliotecarios y altmetrics, hemos realizado una pequeña encuesta online de 11 preguntas, completamente libre y voluntaria, que se encuentra en ANEXO y en el enlace siguiente $<$ http://goo.gl/forms/FdTQJx4xqP>, a los bibliotecarios de universidades españolas, para obtener una visión general de su percepción sin tener en cuenta la biblioteca en la que trabajan y conseguir datos estadísticos sobre la formación específica que han recibido y las tareas que realizan en relación con las altmétricas. Las respuestas se recibieron entre los meses de abril y agosto de 2014. El número de respuestas válidas ha sido de 112, procedentes de 43 bibliotecas universitarias y especializadas.

Los resultados de la encuesta muestran lo siguiente:

1. Sólo en 13 casos han recibido formación sobre altmetrics directamente de su institución; aunque un número considerable (44) se ha auto-formado, lo que es una muestra de su profesionalidad.

2. Veintitrés bibliotecas proporcionan información en su web, un número mayor de las que han formado a sus bibliotecarios, lo que significa que el interés existe aunque no se concrete en pasos más decisivos.

3. Una gran mayoría (90) cree que la biblioteca debe formar o asesorar a sus investigadores en altmetrics. Es rotundo el interés y el compromiso que manifiestan los bibliotecarios con la mejora de la visibilidad de sus investigadores, y ven claro que puede ser interesante esta formación, aunque sea mediante la autoformación previa. 


\section{$¿ C$ Crees que la biblioteca debe formar $o$ asesorar a sus investigadores en altmetrics?}

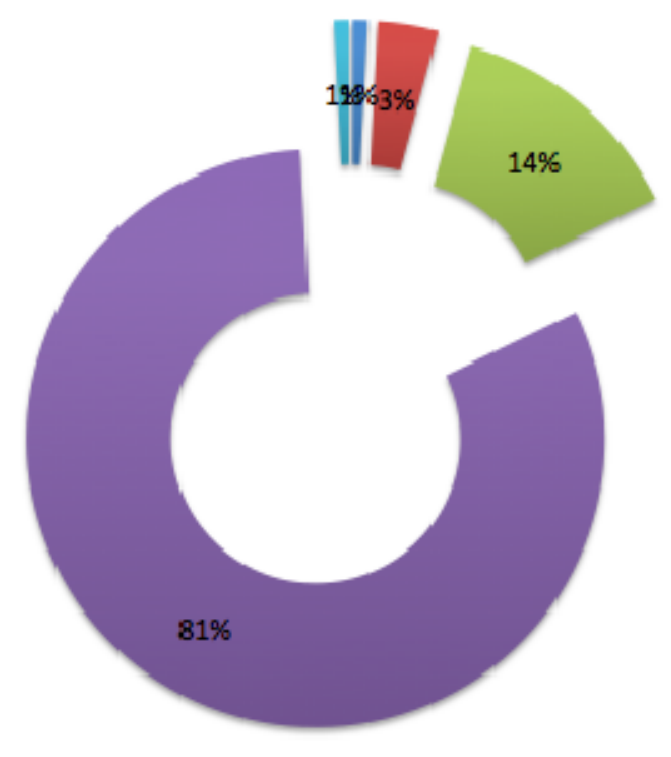

- Informar a través de la web

$=$ NO

No sabe

$\mathrm{nSI}$

Siempre que se considere que pueda serles de utilidad

Figura 1. Respuestas de las bibliotecas a la pregunta sobre la necesidad de formar en altmetrics a sus investigadores.

4. Este último porcentaje se reduce cuando nos movemos de la opinión personal a lo que las bibliotecas han planteado hacer con respecto a la formación o asesoramiento en altmetrics. Aproximadamente la tercera parte de las respuestas son positivas, lo que puede denotar el desconocimiento del tema por una amplia mayoría de bibliotecarios, bibliotecas y universidades, el bajo interés del investigador al no ser consideradas en las evaluaciones oficiales (y en consecuencia el de su institución y de la biblioteca) o a que se está expectante a la espera de que otros den el primer paso.

5. Y cuando se desciende a la práctica nos encontramos con que mayoritariamente no se forma sobre altmetrics en las bibliotecas universitarias españolas, algunas integran algo de información dentro de contenidos más amplios, una de ellas manifiesta que está trabajando en ello, y otra deriva dicha formación al grupo EC3 de Granada. Lo realmente extraño es que exista un número elevado de bibliotecarios que no sepan si su biblioteca ofrece o no algún tipo de formación en altmetrics. ¿Falta de comunicación interna? ¿compartimentos estancos en la biblioteca que hacen desligarse y desconocer lo que hacen los compañeros que se dedican a la formación?...

6. Respecto a proporcionar asesoramiento, el porcentaje de las que lo llevan a cabo es similar al de las que forman, pero aumenta considerablemente el número de respuestas que consideran positivo el asesoramiento. De nuevo la paradoja: se cree conveniente pero no se realiza.

7. Cuando se proporciona alguna formación o asesoría sobre altmetrics a la comunidad universitaria, las bibliotecas coinciden en la formación de tipo más tradicional, como repositorios institucionales, redes sociales y Open Access. La incidencia baja levemente cuando los contenidos hacen referencia a los gestores bibliográficos tipo Mendeley, Google Académico o a redes sociales científicas. La formación que ofrecen desde las bibliotecas apenas incide en temas de marcadores sociales y opciones para compartir en medios sociales o en métodos y servicios alternativos para evaluar las publicaciones científicas.

8. Resulta sorprendente comprobar cómo las tres cuartas partes de respuestas recibidas (Figura 2) creen que altmetrics puede incrementar la visibilidad e impacto de la producción científica o al menos 
complementarla, cuando no se ha generalizado la información, formación y asesoramiento en las Bibliotecas Universitarias Españolas (BUE).

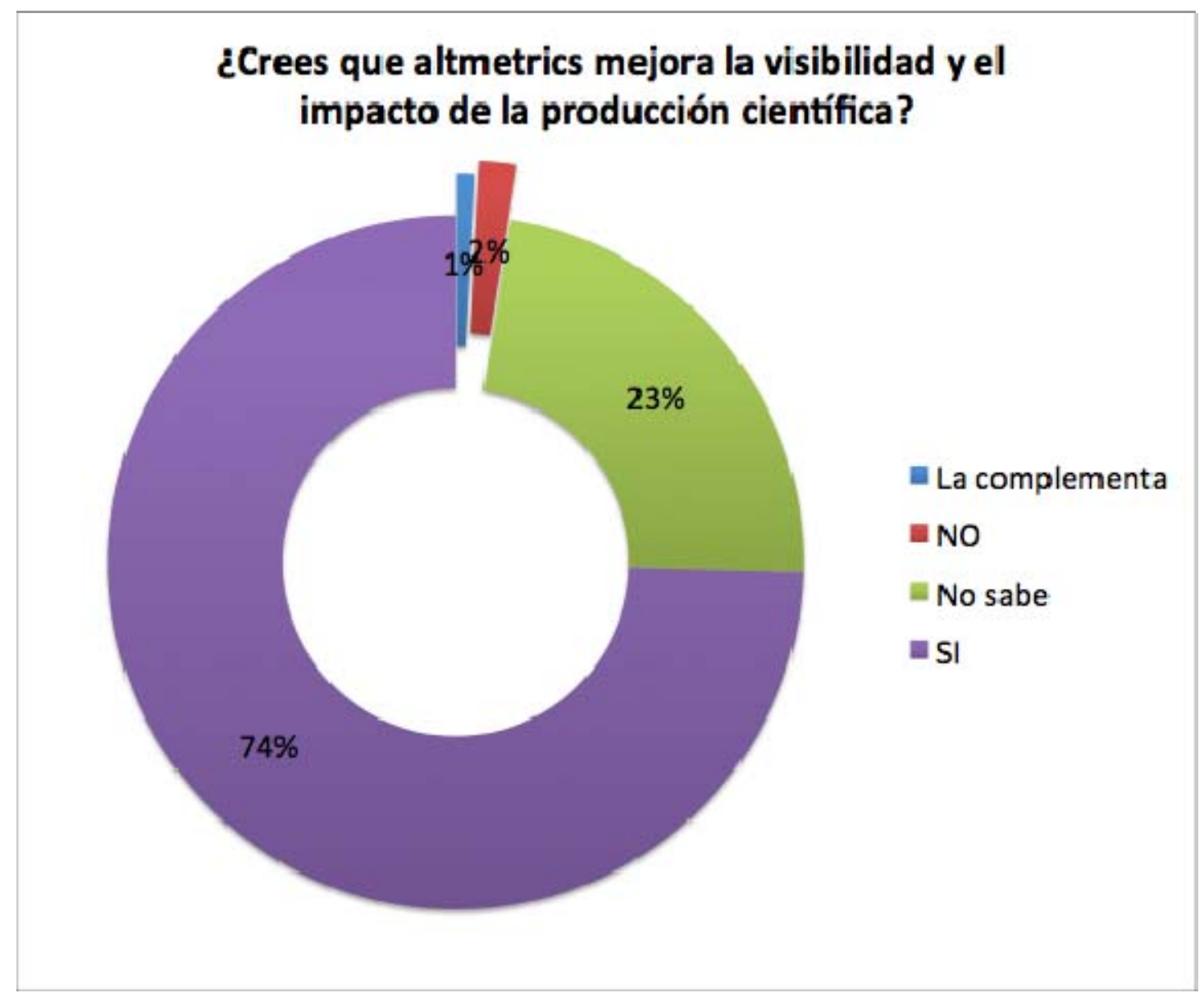

Figura 2. Nivel de opinión de los bibliotecarios españoles en relación al impacto de altmetrics en relación a la visibilidad de la producción científica.

9. Al menos las tres cuartas partes considera que la biblioteca debe colaborar en la evaluación de la investigación de su institución con altmetrics revelando el carácter de servicio que tienen los bibliotecarios, al estar dispuestos a llenar de contenido uno más si la institución así lo requiere.

10. No parece, a tenor de las respuestas de los bibliotecarios que los investigadores conozcan las potenciales ventajas de altmetrics (más del 50\% considera que no lo tienen en cuenta). Además son poquísimos los investigadores que utilizan las redes sociales de investigación (son los más proclives al uso de altmétricas) o al menos existe un desconocimiento del bibliotecario (más del $40 \%$ de las respuestas) sobre el uso de altmetrics por sus investigadores.

11. Las respuestas también proponen líneas de actuación centradas fundamentalmente en la formación de los bibliotecarios como formadores, y de los investigadores, con los que hay que estrechar lazos. La incorporación en los repositorios institucionales y la recopilación de información pertinente y su publicidad en la web son otras de las posibilidades que se apuntan. En los centros en los que por sus características la formación no es posible se sugieren acercamientos prácticos a los investigadores implicados. Alguno manifiesta incluso que está realizando un estudio sobre cómo abordarlo, contemplando todas las cuestiones posibles mencionadas.

\section{CONCLUSIONES}

Las métricas alternativas (altmetrics) o complementarias, son aquellas que contemplan otros intentos de medir, especialmente los vinculados con las redes sociales pero también los que consideran visitas, descargas, usos, relaciones entre investigadores (origen de los índices de citas), las que aparecen en soportes diferentes, las que son 
recopiladas en gestores de información y tal vez un día den como fruto un resultado con impacto. No tienen por qué desplazar a las existentes sino lograr su lugar en un todo analizador, en el que queden al descubierto también sus sesgos, sus fronteras, sus fracasos.

Las bibliotecas, igual que se enfrentan a la marea de información que mina sus mismos fundamentos deben estar preparadas y con una actitud proactiva para apoyar las que demuestren que lo merecen, para ponderar la verdadera dimensión de las candidatas, para ayudar a los investigadores (y ciudadanos) que lo necesiten. Aunque no sean consideradas deben hacer valer su experiencia diaria de trato con la información en todas sus vertientes aprovechando cualquier circunstancia para mostrar su utilidad, impulsándose en las alianzas de los que confían en ella, sabiendo introducirse como solución a necesidades reales, creando herramientas que faciliten la comprensión y la obtención de resultados, formando a quien sea preciso, formándose a sí mismas.

Desde una perspectiva universitaria, estas herramientas enriquecen las funcionalidades de los repositorios, los catálogos de las bibliotecas, sus bases de datos y productos de información aportando información sobre la investigación más popular que está disponible. Sin duda, se trata de una información de utilidad tanto para el investigador como para la biblioteca, que puede recuperar la conversación que surge en la red en torno a la investigación en cuestión. ${ }^{53}$

Su crecimiento es imparable aunque su aplicación generalizada sea menos predecible. Su aceptación o no dependerá de su utilidad y calidad. Los bibliotecarios se encuentran ante la tarea de explicar a sus comunidades la complejidad que supone utilizar estas métricas y la forma de incorporarlas adecuadamente a los sistemas de medición del impacto ya establecidos (Brigham, 2014).

Aún queda camino por recorrer hacia el objetivo de validarlas. Se necesita un mayor compromiso con el uso de los medios sociales y darse cuenta de los beneficios potenciales (Adams y Loach, 2015) ${ }^{54}$, pero lo cierto es que estas herramientas van a ayudar a los investigadores a conocer dónde sus trabajos están siendo discutidos, citados, vistos, guardados, marcados como favoritos y tener una idea del impacto social de su investigación. El papel del bibliotecario en este nuevo escenario cubre un gran abanico por la multiplicidad de frentes en los que se espera mucho de su saber hacer. Se trata de una nueva oportunidad precisamente en unos momentos en los que se replantea su continuidad y en los que se exige la evidencia continua de la rentabilidad de sus actuaciones para sus stakeholders, los investigadores y administradores de sus universidades y centros de investigación, sin olvidar la sociedad en general.

Por lo tanto, el reto es múltiple. Si, como se está demostrando en la literatura profesional, puede ser beneficioso para el investigador y su institución es preciso que sea conocido y valorado por los bibliotecarios; conocido, valorado y utilizado por los investigadores; conocido, valorado e incentivado por la institución y reconocido y valorado por los comités de evaluación de la investigación.

\section{NOTAS}

${ }^{1}$ Así se definieron en la Conferencia de clausura del primer congreso sobre altmetrics <https://www.youtube.com/watch?v=HKbm8Cz54xM>, en la que se establecieron cuáles eran las prioridades en estos momentos: "Definiciones; Identificadores persistentes; Mejora de la calidad de los datos y normalización entre los distintos proveedores; Identificar los tipos de resultados de la investigación aplicables en su mayor parte al uso de métricas; Estandarización de APIs o intercambio de descargas; Proceso de auditoría para la reproducción de datos”.

2 «Alternative Metrics», Nature Materials 11, nº 11 (noviembre de 2012): 907-907, doi:10.1038/nmat3485.

3 Sede de web de ImpactStory <https://impactstory.org/>.

${ }^{4}$ Mike Thelwall - altmetrics, big data, sentiment analysis, webometrics, link analysis, social network sites, blog analysis, methods girl - not to be confused with Michael Thelwell, s. f., <http://www.scit.wlv.ac.uk/ cm1993/mycv.html>.

5 1AM: London - The Altmetrics Conference, s. f., <http://www.altmetricsconference.com/>.

${ }^{6}$ PLOS Article-Level Metrics (ALMs): measuring the impact of research <http://article-level-metrics.plos.org/>.

${ }^{7}$ API de Plum Analytics: <https://github.com/PlumAnalytics/plum-api>.

${ }^{8}$ Noticia de 4 de febrero de 2015: <http://www.altmetric.com/blog/new-source-alert-wikipedia/>.

${ }^{9}$ Altmetric WordPress Plugin: <http://www.altmetric.com/blog/altmetric-wordpress-plugin/>.

${ }^{10}$ Más información en: <http://www.altmetric.com/bookmarklet.php>.

11 Más información en: <http://www.altmetric.com/institutions.php>.

12 Acceso al recurso: <http://www.programmableweb.com/api/citedin>.

13 Acceso al recurso: <http://emdp.elsevier.com/index.asp>.

14 Más información en: <https://www.growkudos.com/>.

${ }^{15}$ NISO Alternative Assessment Metrics Initiative <http://www.niso.org/topics/tl/altmetrics_initiative/>. 

NISO to Develop Standards and Recommended Practices for Altmetrics <http://www.niso.org/news/pr/view?item_key=72efc1097d4caf7b7b5bdf9c54a165818399ec86>.

17 Article-Leval Metrics: <http://sparc.arl.org/initiatives/article-level-metrics> PLOS ONE publications using the PLOS ALM, Mendeley and Altmetric.com. APIs. Figshar.

${ }^{18}$ Según el algoritmo de Altmetric.com. Interactions: november high five: <http://www.altmetric.com/blog/interactions-november-high-five/> .

19 Journals participating in the ScienceDirect pilot: <http://www.altmetric.com/blog/news-roundup-altmetric-in-elsevier-journals-addendum/> .

${ }^{20}$ Ejemplo: el año 2013: <http://www.nature.com/srep/2013/130429/srep01742/full/srep01742.html>.

21 Alternative metrics now available for Cochrane reviews: <http://www.cochranelibrary.com/about/alternative-metrics.html>.

22 Actualización del repositorio e_Buah a la nueva versión 4.1 de $<$ http://www.uah.es/biblioteca/documentos/Actualizacion_\%20repositorio.pdf >.

23 NewBusiness. El mundo empresarial / Noticias: FUTUR, el escaparate de la producción científica de la UPAC, 17 de mayo, 2014. $<$ http://www.elmundoempresarial.es/noticias/es/1405/4/2496/>.

${ }^{24}$ Ranking web de repositorios: <http://repositories.webometrics.info/es/node/24>.

25 Acceso a la noticia: <http://www.urv.cat/es_noticies/341/la-urv-firma-el-segundo-articulo-cientifico-de-mayor-impacto-mundial-en-las-redessociales-en-2013-segun-el-ranking-altmetric>.

${ }^{26}$ OCLC WorldCat Search API Integrado en PlumX Dashboard <http://www.unlimitedpriorities.com/2013/09/oclc-and-plum-analytics/>.

27 Altmetric.com in discovery platforms: <http://www.altmetric.com/blog/discoveryplatforms/>.

${ }^{28}$ Versión institucional de altmetric <http://www.altmetric.com/institutional-edition.php $>$.

${ }^{29}$ Véase un ejemplo en: Bibapp project de MBLWHOI Library <http://research.mblwhoilibrary.org/static/en/trending/trending.html>.

30 Más información en: <http://exchanges.wiley.com/blog/2014/07/08/altmetric-is-now-on-board-for-all-wiley-journals/>. Puede verse un ejemplo en: <http://onlinelibrary.wiley.com/enhanced/doi/10.1002/ece3.1051>.

${ }^{31}$ Más información en: <http://science.okfn.org/2014/05/31/all-metrics-are-wrong-but-some-are-useful/\#sthash.TQt7ULAb.dpuf>.

324 things every librarian should do with altmetrics <http://blog.impactstory.org/4-things-librarians-altmetrics/>.

33 Más información en: <http://www.lib.cuhk.edu.hk/researchcommons/Altmetric\%20CUHK\%201st\%20Session\%20Final.pdf>.

34 Librarians and Research Impact Infografía (Elsevier) <http://libraryconnect.elsevier.com/articles/2014-06/librarians-and-research-impactdownload-and-share-new-infographic-0\#sthash.P72QvRrY.tXWGXqUp.dpuf>.

${ }^{35}$ Sede web de altmetric <http://www.altmetric.com/api-gallery.php>.

${ }^{36}$ Más información en: <https://scholarworks.iu.edu/dspace/>. Ejemplo de guía: <https://blogs.libraries.iub.edu/scholcomm/2014/02/25/socialmedia-metrics-for-iuscholarworks-content-now-available/ $>$.

${ }^{37}$ Caso del acuerdo del Consorcio de Bibliotecas Universitarias Andaluzas (CBUA) y ORCID <http://www.cbua.es/?page_id=957>.

38 Libguides en el mundo anglosajón, que son páginas webs (en algunos casos casi portales). Por ejemplo: University of Pittsburg <http://pitt.libguides.com/c.php?g=12107>; University of Maryland: <http://lib.guides.umd.edu/altmetrics>; Virginia Tech: $<$ http://www.lib.vt.edu/research/metrics/altmetrics/index.html>.

39 University of Leiden: Measuring online impact of research through altmetrics: <http://www.library.leiden.edu/news/altmetrics-weblog.html>; University of Indiana: <https://blogs.libraries.iub.edu/scholcomm/2013/11/25/17-more-essential-altmetrics-resources-the-library-version/>.

40 Stanford University: <http://library.stanford.edu/blogs/stanford-libraries-blog/2014/07/altmetric-workshops-monday-august-4-2014-updatedinfo>; Terceras Jornadas sobre Gestión de la Información Científica (JGIC-2014): <http://bibliotecas.csic.es/widget/inicio/-/contenido/6fd7f0391104-46c5-ad7f-166d9f2c6515;jsessionid=B87ADFC4AB6A524B402F7F16AAC5E4B2>.

41 LIDA 2014: <http://ozk.unizd.hr/lida/theme_two/>. Internet Librarian International $14 \quad<$ http://www.internetlibrarian.com/2014/Wednesday.php> (por celebrarse, en Londres).

42 University of Massachusetts Medical School: <http://libraryguides.umassmed.edu/altmetrics_instruction>. Texas Tech University: <http://library.ttu.edu/news/stories/altmetrics.php>; Canadian Health Libraries Association: <http://www.chla-absc.ca/node/1298>.

${ }^{43}$ Hay varios post en los que Fin Galligan habla de altmetrics. Se pueden ver en un enlace con otras cuestiones en las que también se mencionan las altmetrics < http://www.swets.es/blog/selection-management>. Tiene una serie de 3 posts seguidos dedicados a altmetrics y bibliotecas.

44 Plum Analytics Maps Success in Open Accesss Scholarship: <http://newsbreaks.infotoday.com/NewsBreaks/Plum-Analytics-Maps-Success-inOpen-Access-Scholarship-83478.asp>.

$45<$ http://escholarship.umassmed.edu/jeslib/>.

${ }^{46}$ Puede verse un ejemplo en: <http://research.mblwhoilibrary.org/works/39340>.

${ }^{47}$ Véase un ejemplo en: <http://research.mblwhoilibrary.org/static/en/trending/trending.html>.

${ }^{48}$ Blog LSE. Entrevista a Lucy Ayre. <http://blogs.lse.ac.uk/impactofsocialsciences/2013/08/22/altmetrics-why-the-library-can-embrace-them/>.

49 Más información en las siguientes webs: <http://www.universoabierto.com/7232/altmetrics-evaluacion-del-impacto-de-los-medios-sociales/>, $<$ http://biblioteca.ucm.es/blogs/boletinbibliotecario/8132.php\#.VA8ievl_tuI > o <http://canalbiblos.blogspot.com.es/2014/03/altmetrics-impacto-enla-web.html>.

${ }^{50}$ Más información en las siguientes webs: <http://ddd.uab.cat/record/117830?ln=ca>, <http://euskampus.ehu.es/events/curso-altmetrics/\#\&panel12>, <http://formacionbuva.blogs.uva.es/2014/01/28/webinar-bibliotecarios-y-altmetrics/> o <http://secretariageneral.ugr.es/pages/tablon/*/noticiascanal-ugr/victor-henning-co-fundador-de-la-popular-red-social-mendeley-participa-en-un-seminario-en-la-ugr?lang=en\#.VA8xCfl_tuI>.

51 Más información en: <http://www.slideshare.net/BiblioUCA/ndices-de-citas-y-factor-de-impacto-e-indicios-de-calidad-en-publicacionesacadmicas-para-procesos-de-acreditacin-y-sexenios-para-ciencias-de-la-salud $>\quad$ en: también $<$ http://www.buc.unican.es/sites/default/files/DOCS/guia_herramientas_de_evaluacion_2013.pdf>.

52 Grupo EC3 de la Universidad de Granada <http://seminarioec3.wordpress.com/>.

53 <http://blogs.lse.ac.uk/impactofsocialsciences/2013/08/22/altmetrics-why-the-library-can-embrace-them/>. 
54 Jonathan Adams, jefe científico de Digital Science desde 2013, anteriormente fue director de Research Evaluation for Thomson Reuters (20092013) y Tamar Loach fue invitado como experto en "the European Commission Institute for Prospective Technological Studies (JRC-IPTS) center for a workshop on alternative reputation mechanisms for scholars as a researcher of alternative metrics”.

\section{BIBLIOGRAFÍA}

ACRL Research Planning and Review Committee. Top trends in academic libraries: A review of the trends and issues affecting academic libraries in higher education. College \& Research Library News, 2014, vol. 75, $\mathrm{n}^{\circ}$ 6, junio, p. 294-302. Disponible en: <http://crln.acrl.org/content/75/6/294.full.pdf+html> [Consulta: 8 de marzo de 2015].

ADAMS, J. y LOACH, T. Altmetric mentions and the communication of medical research. Disseminating research outcomes outside academia. [Digital Research Reports]. London, Digital Science, 2015. Disponible en: $<$ http://files.figshare.com/1920206/DRR__Nesta_.pdf> [Consulta: 3 de marzo 2015]

ADIE, E. Gaming altmetrics [Blog Altmetrics], 18 de septiembre de 2013. Disponible en: $<$ http://www.altmetric.com/blog/gaming-altmetrics/> [Consulta: 8 de marzo de 2015].

BANDO, K. When will librarians start research support with altmetrics to their researchers? Now is the time! Figshare, 2013. Disponible en: <http://dx.doi.org/10.6084/m9.figshare.834996>.

BARNES, C. The Use of Altmetrics as a Tool for Measuring Research Impact. Australian Academic \& Research Libraries, abril, 2015, p. 1-14, doi:10.1080/00048623.2014.1003174.

BORREGO, Á. Altmétricas para la evaluación de la investigación y el análisis de necesidades de información. El Profesional de la Información, 2014, vol. 23, nº 4.

BRIGHAM, T.J. An introduction to altmetrics. Medical Reference Services Quarterly, 2014, vol. 33, n 4, p. 43-47, doi:10.1080/02763869.2014.957093.

CARPENTER, T. Lets remove alt from altmetrics [presentación de diapositivas], 31 de marzo de 2015. Disponible en: $<$ http://www.slideshare.net/BaltimoreNISO/carpenter-lets-remove-alt-from-altmetrics-uksg> [Consulta: 8 de abril de 2015].

CHIA, Y.B. y WEE, J. Altmetrics is an Indication of Quality Research or Just HOT Topics. Annual IATUL Conference, 2014, 3 June, Espoo, Finland. Disponible en: <http://docs.lib.purdue.edu/iatul/2014/altmetrics/3/> [Consulta: 19 de septiembre de 2014].

CSIC Abierto. La revista de Digital CSIC, 2013, $\mathrm{n}^{\circ}$ 8, p. $13 . \quad$ Disponible en: <http://digital.csic.es/bitstream/10261/79877/3/CSIC_Abierto_8.pdf> [Consulta: 13 de septiembre de 2014].

DAVIS-KAHLl, S.; FISHELl, T. y KAYE HENSLEY, M. Weaving the threads. Scholarly communication and information literacy. C\&RL News, 2014, vol. 75, nº 8, p. 441-444.

DELASALLE, J. Altmetrics: A librarian’s outlook on potential applications. Library Connect, 2014, mayo, p. 1-4. Disponible en: <http://libraryconnect.elsevier.com/articles/2014-05/altmetrics-librarian-s-outlook-potentialapplications $>$ [Consulta: 8 de marzo de 2015].

DRUMMOND, R. RIMS Revisited: The Evolution of the Research Impact Measurement Service at UNSW Library. Australian Academic \& Research Libraries, 2014, septiembre, p. 1-14, doi: 10.1080/00048623.2014.945065.

GALLIGAN, F. y DYAS-CORREIA, S. Altmetrics: Rethinking the Way We Measure. Serials Review, 2013, vol. 39, $n^{\circ}$ 1, marzo, p. 56-61, doi:10.1080/00987913.2013.10765486.

GALLOWAY, L.M.; PEASE, J.L. y RAUH, A.E. Introduction to Altmetrics for Science, Technology, Engineering, and Mathematics (STEM) Librarians. Science \& Technology Libraries, 2013, vol. 32, $n^{\circ}$ 4, p. 335-345, doi:10.1080/0194262X.2013.829762.

GONZÁLEZ-FERNÁNDEZ-VILLAVICENCIO, N. Altmetrics: sobre bibliotecarios alternativos o alternativa para los bibliotecarios. Nota ThinkEPI, 2015.

GONZÁLEZ-FERNÁNDEZ-VILLAVICENCIO, N. La necesaria confluencia de las competencias informacionales y la comunicación científica. Blok de BiD, 2013, 8 de mayo de 2013 . Disponible en: $<$ http://www.ub.edu/blokdebid/es/content/la-necesaria-confluencia-de-las-competencias-informacionales-y-lacomunicaci\%C3\%B3n-cient\%C3\%ADfica> [Consulta: 8 de marzo de 2015].

GONZÁLEZ MELGOZA, R. Las nuevas herramientas de gestión de la información electrónica y la medición de impacto. [Paper presented at the] 46 Reunión Nacional de Bibliotecarios, 22 - 24 abril, 2014. [ABGRA, Argentina]. Disponible en: <http://www.abgra.org.ar/documentos/46RNB_2014_GonzalezMelgoza.pdf> [Consulta: 8 de marzo de 2015].

GUNN, W. On numbers and freedom. El Profesional de la Información, 2014, vol. 23, nº 5, p. $463-466$.

HOLMES, K. Going beyond bibliometric and altmetric counts to understand impact. Library Connect, 2014. Disponible en: <http://libraryconnect.elsevier.com/articles/2014-05/going-beyond-bibliometric-and-altmetriccounts-understand-impact> [Consulta: 7 de septiembre de 2014]. 
JOHNSON, L. et al. NMC Horizon Report: 2014 Library Edition. Austin, Texas: The New Media Consortium, 2014. Disponible en: <http://cdn.nmc.org/media/2014-nmc-horizon-report-library-EN.pdf> [Consulta: 15 de diciembre de 2014].

KING, P. y THUNA, M. Altmetrics in context, 2013. Canadian Association of Research Libraries=Association des bibliothèques de recherche du Canada. Disponible en: <http://www.carl-abrc.ca/uploads/SCC/CARL2013altmetrics-EN-FA.pdf> [Consulta: 8 de marzo de 2015].

KONKIEL, S. Altmetrics: A 21st Century Solution to Determining Research Quality. Online Searcher, 2013, julio/agosto, p. 10-16. Disponible en: <https://scholarworks.iu.edu/dspace/handle/2022/17147> [Consulta: 20 de febrero de 2014].

KONKIEL, S. y SCHERER, D. New Opportunities for Repositories in the Age of Altmetrics. Bulletin ASIS\&T. The Information Association for the Information Age, 2013, abril/mayo. Disponible en: <https://asis.org/Bulletin/Apr13/AprMay13_Konkiel_Scherer.html> [Consulta: 27 de septiembre de 2014].

KWOK, R. Research impact: Altmetrics make their mark. Nature, 2013, nº 500, p. 491-493. doi:10.1038/nj7463-491a.

LAPINSKI, S.; PIOWOWAR, H. y PRIEM, J. Riding the crest of the altmetrics wave: How librarians can help prepare faculty for the next generation of research impact metrics. News College \& Research Libraries. ACRL, 2013, vol. 74, $n^{\circ}$ 6, p. 292-300.

MERLO-VEGA, J.-A. Ciencia 2.0: la aportación de las bibliotecas universitarias. Anuario ThinkEPI, 2011, $\mathrm{n}^{0}$ 5, p. 155-161. Disponible en: <http://www.thinkepi.net/ciencia-dospuntocero-la-aportacion-de-las-bibliotecasuniversitarias $>$ [Consulta: 18 de octubre de 2014].

NISO. Altmetrics Standards Project White Paper, Version 4 - draft for public comment through, 2014,18 julio. Disponible en: <http://www.niso.org/apps/group_public/document.php?document_id=13295\&wg_abbrev=altmetrics> [Consulta: 18 de octubre de 2014].

NOORDEN, R. Scientistis and the social network. Nature, 2014, vol. 512, $\mathrm{n}^{\mathrm{o}}$ 14, p. 126-129. Disponible en: <http://www.nature.com/news/online-collaboration-scientists-and-the-social-network-1.15711> [Consulta: 15 de febrero de 2015].

PETERS, I. et al. Research Data Explored: Citations versus Altmetrics, 2015. Disponible en: $<$ http://arxiv.org/ftp/arxiv/papers/1501/1501.03342.pdf> [Consulta: 15 de febrero de 2015].

PRIEM, J.; GROTH, P. y TARABORELLI, D. The Altmetrics Collection. PLOS ONE, 2012, vol. 7, n 11 (1 de noviembre de 2012), doi:10.1371/journal.pone.0048753.

PRIEM, J.; PIWOWAR, H. y HEMMINGER, B.M. Altmetrics in the wild: Using social media to explore scholarly impact. arXiv:1203.4745 [cs], 20 de marzo de 2012. Disponible: <http://arxiv.org/abs/1203.4745> [Consulta: 12 de febrero de 2014].

PRIEM, J. et al. Altmetrics: a manifesto, 2012. Disponible en: <http://altmetrics.org/manifesto/> [Consulta: 12 de febrero de 2014].

PROCTER, R. et al. Adoption and use of Web 2.0 in scholarly communications. Philosophical Transactions of the Royal Society A: Mathematical, Physical and Engineering Sciences, 2010, vol. 368, $\mathrm{n}^{\circ} 1926$ (2 de agosto de 2010), doi:10.1098/rsta.2010.0155.

RATHEMACHER, A.J. Altmetrics: Help Your Researchers Measure Their Full Impact. Serials Review, 2014, vol. 40, $\mathrm{n}^{\circ} 1$, p. 60-64.

RODGERS, E.P. y BARBROW, S. A Look at Altmetrics and Its Growing Significance to Research Libraries, 2013. The University of Michigan University Library. [Deep Blue Repository]. Disponible en: <http://deepblue.lib.umich.edu/handle/2027.42/99709> [Consulta: 8 de marzo de 2015].

ROEMER, R.C. y BORCHARDT, R. From Bibliometrics to Altmetrics A Changing Scholarly Landscape. College \& Research Libraries News, 2012, vol. 73, $\mathrm{n}^{0}$ 10, p. 596-600. Disponible en: $<$ http://crln.acrl.org/content/73/10/596.full.pdf+html> [Consulta: 19 de febrero de 2014].

ROEMER, R.C. y BORCHARDT, R. Institutional Altmetrics and Academic Libraries. Information Standards Quarterly, 2013, vol. 25, n² 2, p. 14-19.

ROEMER, R.C. y BORCHARDT, R. Keeping Up With... Altmetrics. ACRL Keeping up with..., 2014. Disponible en: <http://www.ala.org/acrl/publications/keeping_up_with/altmetrics> [Consulta: 18 de octubre de 2014].

RONALD, R. y FRED Y.Y. A multi-metric approach for research evaluations. Chinese Science Bulletin, 2013 , vol. 58, n 26, p. 3288-3290, doi: 10.1007/s11434-013-5939-3.

SUD, P. y THELWALL, M. Evaluating altmetrics. Scientometrics, 2014, vol. 98, no 2, p. 1131-1143, doi: 10.1007/s11192-013-1117-2.

SUTTON, S.W.H. Altmetrics: What Good are They to Academic Libraries? Kansas Library Association College and University Libraries Section Proceedings, 2014, vol. 4, n 2.

TANANBAUM, G. Article-Level Metrics: A SPARC Primer, 2013. Disponible en: <http://www.sparc.arl.org/sites/default/files/sparc-alm-primer.pdf> [Consulta: 18 de octubre de 2014]. 
TORRES-SALINAS, D. y CABEZAS-CLAVIJO, A. Altmetrics: no todo lo que se puede contar, cuenta. Anuario ThinkEPI, 2013, vol. 7, p. 114-117. Disponible en: <http://digibug.ugr.es/bitstream/10481/26361/1/114-117Torres-Salinas-Cabezas-Almetrics.pdf > [Consulta: 8 de marzo de 2015].

ZAHEDI, Z.; FENNER, M. y COSTAS, R. "How consistent are altmetrics providers? Study of 1000 PLOS ONE publications using the PLOS ALM, Mendeley and Altmetric.com APIs", Figshare, 2014. Disponible en: $<$ http://dx.doi.org/10.6084/m9.figshare.1041821>.

YOU, J. Who are the science stars of Twitter? Science communicators and some highly cited research, but few women, make top 50 list. Science, 2014, vol. 345, nº 6203. 


\section{ANEXO}

Encuesta dirigida a los bibliotecarios de universidades españolas sobre temas relacionados con altmetrics. Se encuentra también en el siguiente enlace: <http://goo.gl/forms/FdTQJx4xqP>.

Como profesional, ¿has recibido formación sobre altmetrics a través de tu institución? *

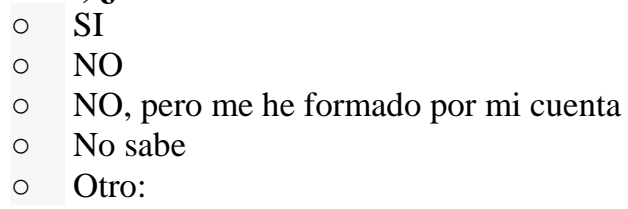

¿La web de la biblioteca ofrece información sobre algún tema relacionado con altmetrics? *

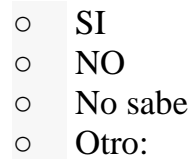

¿Crees que la biblioteca debe formar o asesorar a sus investigadores en altmetrics? *

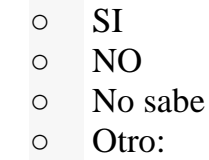

¿Se ha planteado tu biblioteca ofrecer formación o asesoramiento en altmetrics? *

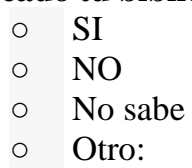

¿Imparte tu biblioteca algún tipo de formación en altmetrics? *

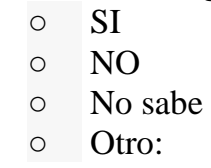

¿Ofrece tu biblioteca algún tipo de asesoramiento en altmetrics? *

$\begin{array}{ll}\circ & \text { SI } \\ \circ & \text { NO } \\ \circ & \text { No sabe } \\ \circ & \text { Otro: }\end{array}$

En el caso de incluir alguna formación o asesoría sobre altmetrics a la comunidad universitaria, ¿qué contenidos se tratan? *

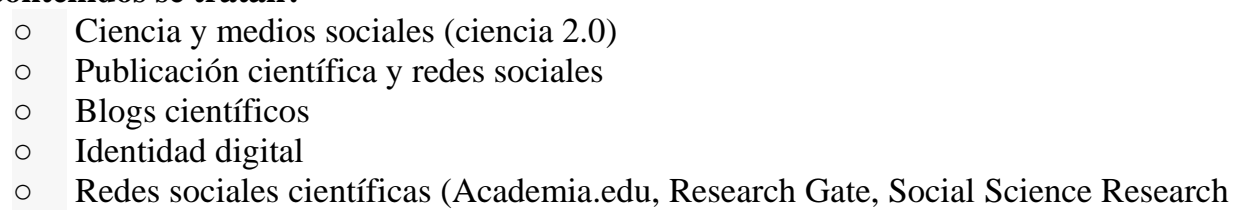
Network (SSRN), etc.,)

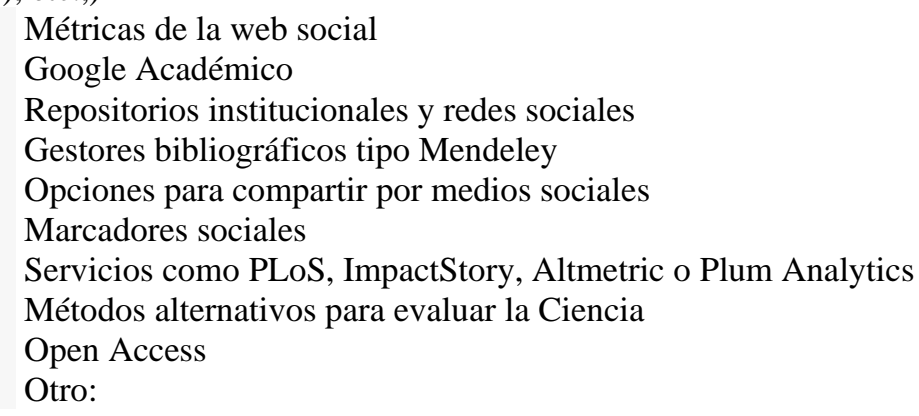


¿Crees que altmetrics mejora la visibilidad y el impacto de la producción científica? *

$$
\begin{array}{ll}
\circ & \text { SI } \\
\circ & \text { NO } \\
\circ & \text { No sabe } \\
\circ & \text { Otro: }
\end{array}
$$

¿Consideras que la biblioteca debe colaborar en la evaluación de la investigación de su institución con altmetrics? *
- SI
- NO
- No sabe
- Otro:

¿Crees que los investigadores de tu institución tienen en cuenta altmetrics? *

$$
\begin{array}{ll}
\circ & \text { SI } \\
\circ & \text { NO } \\
\circ & \text { No sabe } \\
\circ & \text { Otro: }
\end{array}
$$

¿Propondrías alguna línea de actuación para incorporar altmetrics en el trabajo de los bibliotecarios?

Nombre de la Institución en la que trabajas *

Añade el comentario que quieras 